\section{Ausschreibung der Forschungspreise der Deutschen Gesellschaft für Pneumologie und Beatmungsmedizin e.v.}

Die Deutsche Gesellschaft für Pneumologie und Beatmungsmedizin e.V. (DGP) schreibt zwei Forschungspreise aus. Die Preise dienen der Förderung des wissenschaftlichen Nachwuchses in der Pneumologie. Sie werden für die beste grundlagenwissenschaftliche und die beste klinische Arbeit aus dem Gesamtgebiet der Pneumologie verliehen und sind mit je $€ 10.000$,dotiert.

Die eingereichte Arbeit muss in deutscher oder englischer Sprache verfasst und darf zu keinem anderen Preis eingereicht sein. Ist die Arbeit bereits publiziert, darf das Erscheinungsdatum der Publikation nicht mehr als ein Jahr vor dem Abgabetermin zur Preiseinreichung liegen. Mehrere thematisch zusammenhängende Publikationen können zusammengefasst und - mit einer ein- bis zweiseitigen Zusammenfassung versehen eingereicht werden. Sollte die Arbeit in einer Forschergruppe erstellt oder die Bewerberin nicht Erstautorin/der Bewerber nicht Erstautor einer zugehörigen Publikation sein, bitten wir um genaue Angabe des Eigenanteils.
Die Bewerberinnen und Bewerber sollen zum Zeitpunkt der Bewerbungsfrist nicht über 40 Jahre alt sein. Sie müssen ihre $\mathrm{Ar}$ beitsstätte in Deutschland haben. Wenn die eingereichten Forschungsarbeiten in Deutschland ausgeführt wurden, wird dies ebenfalls berücksichtigt.

Beide Preise werden auf der Eröffnungsveranstaltung des DGP-Kongresses am 14. März 2019 in München verliehen und es wird erwartet, dass die Preise persönlich entgegengenommen werden.

Um sich zu bewerben, senden Sie Ihre Arbeit bitte bis zum 8. Januar 2019 im PdfFormat (max. 5MB) mit einer Publikationsliste und Ihrem Lebenslauf an den Generalsekretär der Deutschen Gesellschaft für Pneumologie und Beatmungsmedizin, Prof. Dr. Winfried J. Randerath (info@pneumologie.de). Unvollständige Bewerbungen werden nicht berücksichtigt. Die Entscheidung des Auswahlkomitees ist nicht anfechtbar.

Prof. Dr. Klaus F. Rabe (Präsident)

Prof. Dr. Winfried J. Randerath

(Generalsekretär) 